\title{
Operators on Manifolds with Conical Singularities
}

\author{
L. Ma and B.-W. Schulze
}

July 7,2009

\begin{abstract}
We construct elliptic elements in the algebra of (classical pseudo-differential) operators on a manifold $M$ with conical singularities. The ellipticity of any such operator $A$ refers to a pair of principal symbols $\left(\sigma_{0}, \sigma_{1}\right)$ where $\sigma_{0}$ is the standard (degenerate) homogeneous principal symbol, and $\sigma_{1}$ is the so-called conormal symbol, depending on the complex Mellin covariable $z$. The conormal symbol, responsible for the conical singularity, is operator-valued and acts in Sobolev spaces on the base $X$ of the cone. The $\sigma_{1}$-ellipticity is a bijectivity condition for all $z$ of real part $(n+1) / 2-\gamma, n=\operatorname{dim} X$, for some weight $\gamma$. In general, we have to rule out a discrete set of exceptional weights that depends on $A$. We show that for every operator $A$ which is elliptic with respect to $\sigma_{0}$, and for any real weight $\gamma$ there is a smoothing Mellin operator $F$ in the cone algebra such that $A+F$ is elliptic including $\sigma_{1}$. Moreover, we apply the results to ellipticity and index of (operator-valued) edge symbols from the calculus on manifolds with edges.
\end{abstract}

\section{Mathematics Subject Classification:}

Primary: 35S35

Secondary: 35J70

Keywords: Operators on manifolds with conical singularities, conormal symbols, ellipticity of cone operators, parametrices and invertibility within the cone calculus

\section{Contents}

Introduction

1 Operators in the Cone Algebra 2

2 Ellipticity with Respect to Prescribed Weights $\quad 6$

3 The Case of Manifolds with Conical Singularities and Boundary 9

4 Some Consequences for Edge Symbols $r 12$

$\begin{array}{lr}\text { References } & 14\end{array}$

\section{Introduction}

Elliptic (and other types) of operators on a manifold with conical singularities have been studied for a long time, both from the point of view of regularity and asymptotics of solutions in weighted Sobolev spaces, cf. Kondratyev [5] (for the case of boundary value problems), Melrose, Mendoza [6], Rempel, Schulze [?], and spectral geometry, index theory, or topology, cf. Cheeger [3], or Shaw [11] (more references will be given below). Manifolds with conical singularities belong to a hierarchy of categories of stratified spaces, containing manifolds with (smooth) boundary, or 
edge, corner, or higher polyhedral singularities (briefly referred to as manifolds with singularities). Locally, such spaces are obtained by iteratively forming cones and wedges (starting from smooth manifolds as bases of cones). Corresponding algebras of operators referring to the stratification also have an iterative structure, cf. [9], [10]. Ellipticity of an operator $A$ on a space of singularity order $k \in \mathbb{N}$ (where $k=0$ corresponds to smoothness, $k=1$ to conical or edge singularities) is a bijectivity condition on the components of a principal symbolic hierarchy $\sigma=\left(\sigma_{j}\right)_{j=0, \ldots, k}$. It is interesting for many reasons to construct elliptic operators in that sense, starting from a given operator that is only elliptic with respect to $\sigma_{0}$, the standard homogeneous principal symbol on the main stratum. Answers in general may be very complicated. In the case of conical singularities we show the existence of a smoothing (in general non-compact) Mellin operator $F$ belonging to the cone algebra such that $A+F$ is also elliptic with respect to $\sigma_{1}$ for a prescribed weight $\gamma \in \mathbb{R}$ (where, of course, $\sigma_{0}(A+F)=\sigma_{0}(A)$ ). As we shall see below, such a result considerably simplifies the parametrix construction in the cone algebra. Other applications concern boundary symbols of operators on a manifold with boundary (or, more generally, edge symbols on a manifold with edge). Note that smoothing Mellin operators with the claimed properties in the case of a smooth and closed base can also be deduced from the results of Witt [12]. It is difficult to predict to what extent this approach works in other cases, for instance, boundary value problems. In any case, our construction is relatively elementary and apparently rather general.

Our result concerns the general nature of pseudo-differential algebras on a manifold with singularities. The presence of symbolic hierarchies is a new aspect, compared with the smooth case. It is, of course, desirable that the construction of elliptic elements is not too problematic. However, it is hard to decide whether ellipticity of conormal symbols is possible for a weight $\gamma$ when the non-smoothing part $A_{0}$ of a $\sigma_{0}$-elliptic operator is given. This depends on the individual operator and requires information on "non-linear eigenvalues" contained in the conormal symbols; those usually rule out a discrete set of weights. We construct here a new variety of explicit examples of $\left(\sigma_{0}, \sigma_{1}\right)$-elliptic elements in the cone calculus, no matter what $A_{0}$ and $\gamma$ exactly are.

Acknowledgement: This paper has been produced during a working stay at the Chern Institute of Mathematics at the Nankai University in Tianjin, PR China, and the authors thank Professor Long Yiming for the generous support.

\section{Operators in the Cone Algebra}

Let $M$ be a manifold with conical singularities $v_{1}, \ldots, v_{N}$. For simplicity we consider the case $N=1$, and set $v:=v_{1}$ (the case of arbitrary $N$ is similar and left to the reader). If $X$ is a closed $C^{\infty}$ manifold, the cone $X^{\Delta}:=\left(\overline{\mathbb{R}}_{+} \times X\right) /\left(\mathbb{R}_{+} \times X\right)$ is an example of such an $M$; in this case the conical singularity is represented by $\{0\} \times X$ in the quotient space. In general $M$ is locally near $v$ modelled on such a cone. More precisely, $M \backslash\{v\}$ is smooth, and we have a "singular chart"

$$
\chi: V \rightarrow X^{\Delta}
$$

for some neighbourhood $V$ of $v$ in $M$ and a smooth manifold $X=X(v)$ where $\chi(v)$ is equal to the vertex of $X^{\Delta}$, and $\chi_{\mathrm{reg}}:=\left.\chi\right|_{V \backslash\{v\}}: V \backslash\{v\} \rightarrow X^{\wedge}:=\mathbb{R}_{+} \times X$ is a diffeomorphism. If $\tilde{\chi}: V \rightarrow X^{\Delta}$ is another such map, then $\tilde{\chi}_{\text {reg }} \circ \chi_{\text {reg }}^{-1}: X^{\wedge} \rightarrow X^{\wedge}$ is asked to be the restriction of a diffeomorphism $\mathbb{R} \times X \rightarrow \mathbb{R} \times X$ to $X^{\wedge}$.

Let Diffm $m$ deg $(M)$ denote the set of all differential operators on $M \backslash\{v\}$ with smooth coefficients that are locally near $v$ in the splitting of variables $(r, x) \in X^{\wedge}$ (coming from $\chi_{\text {reg }}$ ) of the form

$$
A=r^{-m} \sum_{j=0}^{m} a_{j}(r)\left(-r \partial_{r}\right)^{j}
$$

for coefficients $a_{j} \in C^{\infty}\left(\overline{\mathbb{R}}_{+}, \operatorname{Diff}^{m-j}(X)\right)$. Here $\operatorname{Diff}^{k}(\cdot)$ means the space of all differential operators of order $k$ on the manifold in parentheses. 
By the cone algebra on $M$ we understand an algebra of classical pseudo-differential operators on $M \backslash\{v\}$ that contains $\operatorname{Diff}_{\mathrm{deg}}^{m}(M)$ together with the parametrices of elliptic elements. The ellipticity refers to a pair $\left(\sigma_{0}, \sigma_{1}\right)$ of principal symbols. For $A \in \operatorname{Diff}_{\operatorname{deg}}^{m}(M)$ those are defined as follows. The component $\sigma_{0}(A)$ is the standard homogeneous principal symbol of $A$ as a function in $C^{\infty}\left(T^{*}(M \backslash\{v\}) \backslash 0\right)$. In the splitting of variables $(r, x)$ with $x$ varying in an open set of $\mathbb{R}^{n}, n=\operatorname{dim} X$, it has the form

$$
\sigma_{0}(A)(r, x, \rho, \xi)=r^{-m} \tilde{\sigma}_{0}(A)(r, x, r \rho, \xi)
$$

for the corresponding covariables $(\rho, \xi)$, and a function $\tilde{\sigma}_{0}(A)(r, x, \tilde{\rho}, \xi)$ which is smooth up to $r=0$. To recall also the definition on $\sigma_{1}(A)$ we fix some notation on pseudo-differential operators on a $C^{\infty}$-manifold $X$. The corresponding tools will be necessary anyway for the constructions below. We need the operators in parameter-dependent form with a parameter $\lambda \in \mathbb{R}^{l}$ for some $l \in \mathbb{N}$ for $\mathbb{N}=\{0,1,2, \ldots\}$. Locally in an open set in $\mathbb{R}^{n}$ those operators are defined in terms of classical symbols $a(x, \xi, \lambda)$ of order $m$ where $(\xi, \lambda)$ are treated as covariables, and the operators in $x$ are expressed via the Fourier transform. Here "classical" means that $a(x, \xi, \lambda)$ has an asymptotic expansion in functions that are homogeneous of order $m-j, j \in \mathbb{N}$ for $|\xi, \lambda| \geq$ const for a constant $>0$. Based on an open covering of $X$ by charts and a subordinate partition of unity we obtain associated pseudo-differential operators, depending on $\lambda$. The space $L_{\mathrm{cl}}^{m}\left(X ; \mathbb{R}^{l}\right)$ is defined to be the set of those operator families, plus smoothing families that belong to $\mathcal{S}\left(\mathbb{R}^{l}, L^{-\infty}(X)\right)$. Let

$$
\Gamma_{\beta}:=\{z \in \mathbb{C}: \operatorname{Re} z=\beta\}
$$

for any $\beta \in \mathbb{R}$, and denote by $L_{\mathrm{cl}}^{m}\left(X ; \Gamma_{\beta}\right)$ the space of all operator fuctions $a(z)$ depending on $z \in \Gamma_{\beta}$ such that $a(\beta+i \rho) \in L_{\mathrm{cl}}^{m}(X ; \mathbb{R})$.

For $A \in \operatorname{Diff}_{\mathrm{deg}}^{m}(M)$, locally near $v$ written in the form (1.2) we set

$$
\sigma_{1}(A)(z):=\sum_{j=0}^{m} a_{j}(0) z^{j}: H^{s}(X) \rightarrow H^{s-m}(X)
$$

here $X$ is assumed to be compact. In other words, we interpret $\sigma_{1}(A)(z)$ as a family of operators on the base $X$ of the cone, acting on Sobolev spaces of smoothness $s$. We have $\left.\sigma_{1}(A)\right|_{\Gamma_{\beta}} \in L_{\mathrm{cl}}^{m}\left(X ; \Gamma_{\beta}\right)$ for every $\beta \in \mathbb{R}$. Let us call the operator $\sigma_{0}$-elliptic, if $\sigma_{0}(A)$ does not vanish on $T^{*}(M \backslash\{v\}) \backslash 0$, and if the function $\tilde{\sigma}_{0}(A)(r, x, \tilde{\rho}, \xi)$ is non-vanishing up to $r=0$. Moreover, $A$ is called $\sigma_{1}$-elliptic with respect to a weight $\gamma \in \mathbb{R}$ if (1.3) is a family of isomorphisms for all $z \in \Gamma_{(n+1) / 2-\gamma}$ and some $s \in \mathbb{R}$.

Let us now recall some well-known consequences of the $\sigma_{0}$-ellipticity of $A$. In general, an operator family in $L_{\mathrm{cl}}^{m}\left(X ; \mathbb{R}^{l}\right)$ is called parameter-dependent elliptic (of order $m$ ) if its homogeneous principal symbol in $(\xi, \lambda)$ is non-vanishing for all $(\xi, \lambda) \neq 0$. In particular, we have the notion of parameterdependent ellipticity in $L_{\mathrm{cl}}^{m}\left(X ; \Gamma_{\beta}\right)$. Let $A \in \operatorname{Diff}_{\mathrm{deg}}^{m}(M)$ be $\sigma_{0}$-elliptic; then $\sigma_{1}(A)(z) \in L_{\mathrm{cl}}^{m}\left(X ; \Gamma_{\beta}\right)$ is parameter-dependent elliptic for every fixed $\beta \in \mathbb{R}$. This property is uniform (in an obvious sense) with respect to $\beta=\operatorname{Re} z$ in compact intervals. Thus, from the fact that a parameter-dependent elliptic family induces isomorphisms $H^{s}(X) \rightarrow H^{s-m}(X)$ when the absolute value of the parameter is sufficiently large (which is in our case uniform in compact $\beta$-intervals) it follws that there is a non-empty set $E \subset \mathbb{C}$ such that (1.3) are isomorphisms for all $z \in E$. Moreover, since (1.3) is a holomorphic family of Fredholm operators, there is a discrete set $D \subset \mathbb{C}$ such that (1.3) are isomorphisms for all $z \in \mathbb{C} \backslash D$. In particular, we have $E=\mathbb{C} \backslash D$, and $D \cap\left\{c_{1} \leq \operatorname{Re} z \leq c_{2}\right\}$ is finite for every $c_{1} \leq c_{2}$.

Define $\mathcal{H}^{s, \gamma}\left(X^{\wedge}\right)$ as the set of all $u \in H_{\text {loc }}^{s}\left(X^{\wedge}\right)$ such that

$$
(2 \pi i)^{-1} \int\left\|R^{s}\left(z, \lambda^{1}\right) M_{\gamma-n / 2} u(z)\right\|_{L^{2}(X)}^{2} d z<\infty
$$

where $R^{s}(z, \lambda) \in L_{\mathrm{cl}}^{s}\left(X ; \Gamma_{(n+1) / 2-\gamma} \times \mathbb{R}^{l}\right)$ is some parameter-dependent family, elliptic of order $s$, and $\lambda^{1} \in \mathbb{R}^{l}$ sufficiently large. Moreover, $M_{\gamma-n / 2}$ is the weighted Mellin transform, first defined on 
$C^{\infty}\left(\mathbb{R}_{+}, C^{\infty}(X)\right)$ by the formula $M_{\gamma-n / 2} u(z)=\left.\int r^{z-1} u(r) d r\right|_{\operatorname{Re} z=(n+1) / 2-\gamma}$, and then extended to $u \in \mathcal{H}^{s, \gamma}\left(X^{\wedge}\right)$. This yields an isomorphism $M_{\gamma-n / 2}: \mathcal{H}^{s, \gamma}\left(X^{\wedge}\right) \rightarrow \hat{H}^{s}\left(\Gamma_{(n+1) / 2-\gamma} \times X\right)$ where $\hat{H}^{s}\left(\Gamma_{\beta} \times X\right)$ is the image of the cylindrical Sobolev space $H^{s}(\mathbb{R} \times X)$ under the one-dimensional Fourier transform $v(t, x) \rightarrow \int e^{-i t \rho} v(t, x) d t$ with $\rho$ being interpreted as $\operatorname{Im} z$ for $z \in \Gamma_{\beta}$. Globally on a compact manifold $M$ with conical singularity $v$ the weighted space $\mathcal{H}^{s, \gamma}(M)$ is defined to be the set of all $u \in H_{\mathrm{loc}}^{s}(M \backslash\{v\})$ such that $\omega \chi_{*} u \in \mathcal{H}^{s, \gamma}\left(X^{\wedge}\right)$ where $\chi_{*}$ is the push forward under (1.1) and $\omega$ a cut-off function on the half-axis (i.e., $\omega \in C_{0}^{\infty}\left(\overline{\mathbb{R}}_{+}\right), \omega=1$ close to 0$)$. It can easily be verified that an $A \in \operatorname{Diff}_{\mathrm{deg}}^{m}(M)$ induces continuous operators

$$
A: \mathcal{H}^{s, \gamma}(M) \rightarrow \mathcal{H}^{s-m, \gamma-m}(M)
$$

for all $s, \gamma \in \mathbb{R}$. Operators $A \in \operatorname{Diff}_{\mathrm{deg}}^{m}(M)$, or, more generally, pseudo-differential operators in the cone calculus of [7] will also be referred to as operators in the cone algebra. Some elements of the theory will be outlined below. Close to $v$ they are formulated in terms of meromorphic operator-valued symbols. Later on we also need the spaces

$$
\mathcal{K}^{s, \gamma}\left(X^{\wedge}\right)=\left\{\omega u_{0}+(1-\omega) u_{\infty}: u_{0} \in \mathcal{H}^{s, \gamma}\left(X^{\wedge}\right), u_{\infty} \in H_{\text {cone }}^{s}\left(X^{\wedge}\right)\right\}
$$

where $\omega$ is any cut-off function, and $(1-\omega(r)) H_{\text {cone }}^{s}\left(X^{\wedge}\right)=(1-\omega(|\tilde{x}|)) H^{s}\left(\mathbb{R}_{\tilde{x}}^{n+1}\right)$ in the case $X=S^{n}$ (the unit sphere in $\mathbb{R}_{\tilde{x}}^{n+1}$ ); for general $X$ we refer to a definition via the latter representation, localised in conical subsets in $\mathbb{R}_{\tilde{x}}^{n+1} \backslash\{0\} \cong \mathbb{R}_{+} \times U$ for coordinate neighbourhoods $U$ on $X$.

If $E$ is a Fréchet space and $U \subseteq \mathbb{C}$ open then $\mathcal{A}(U, E)$ denotes the space of all holomorphic functions on $U$ with values in $E$. Applying that to $E=L_{\mathrm{cl}}^{m}\left(X ; \mathbb{R}^{l}\right.$ ) (first for $l=0$ ) we obtain the space $M_{\mathcal{O}}^{m}(X)$, defined as the subspace of all $h(z) \in \mathcal{A}\left(\mathbb{C}, L_{\mathrm{cl}}^{m}(X)\right)$ such that $\left.h\right|_{\Gamma_{\beta}} \in L_{\mathrm{cl}}^{m}\left(X ; \Gamma_{\beta}\right)$ for every $\beta \in \mathbb{R}$, uniformly in compact $\beta$-intervals. Also $M_{\mathcal{O}}^{m}(X)$ is Fréchet in a natural way; this allows us to form the spaces $C^{\infty}\left(\overline{\mathbb{R}}_{+} \times \overline{\mathbb{R}}_{+}, M_{\mathcal{O}}^{m}(X)\right)$. For instance, we have $h(r, z):=\sum_{j=0}^{m} a_{j}(r) z^{j} \in$ $C^{\infty}\left(\overline{\mathbb{R}}_{+}, M_{\mathcal{O}}^{m}(X)\right)$, cf. the notation in $(1.2)$, and $A=r^{-m}$ op $_{M}^{\delta}(h)$ where op ${ }_{M}^{\delta}(\cdot)$ means the pseudodifferential operator based on the weighted Mellin transform, of weight $\delta \in \mathbb{R}$, namely,

$$
\mathrm{op}_{M}^{\delta}(h) u(r)=\iint\left(r / r^{\prime}\right)^{-(1 / 2-\delta+i \rho)} h\left(r, r^{\prime}, 1 / 2-\delta+i \rho\right) u\left(r^{\prime}\right) d r^{\prime} / r^{\prime} d \rho
$$

$₫ \rho=(2 \pi)^{-1} d \rho$, here formulated for an $\left(r, r^{\prime}\right)$-dependent Mellin amplitude function $h$. Recall that the expression (1.5) makes sense as an oscillatory integral, first for $u \in C_{0}^{\infty}\left(\mathbb{R}_{+}, C^{\infty}(X)\right)$ and induces a continuous operator $C_{0}^{\infty}\left(\mathbb{R}_{+}, C^{\infty}(X)\right) \rightarrow C^{\infty}\left(\mathbb{R}_{+}, C^{\infty}(X)\right)$. Clearly, (1.5) represents a standard pseudo-differential operator on $X^{\wedge}$, however, in the Mellin operator convention. The operator $r^{-m} \operatorname{op}_{M}^{\gamma-n / 2}(h)$ extends to a continuous operator

$$
r^{-m} \operatorname{op}_{M}^{\gamma-n / 2}(h): \mathcal{H}^{s, \gamma}\left(X^{\wedge}\right) \rightarrow \mathcal{H}^{s-m, \gamma-m}\left(X^{\wedge}\right)
$$

when $h$ is independent of $r, r^{\prime}$ for large $r, r^{\prime}$, for arbitrary $\gamma \in \mathbb{R}$. Operators of the form (1.6) belong to the ingredients of the cone algebra. Other operators are smoothing close to the tip (although not necessarily compact), especially, smoothing Mellin operators with meromorphic symbols. Let us call a sequence

$$
R=\left\{\left(r_{j}, n_{j}\right)\right\}_{j \in \mathbb{Z}} \subset \mathbb{C} \times \mathbb{N}
$$

a Mellin asymptotic type, when $\pi_{\mathbb{C}} R:=\left\{r_{j}\right\}_{j \in \mathbb{Z}}$ intersects every strip $c \leq \operatorname{Re} z \leq c^{\prime}$ in a finite set, for every $c \leq c^{\prime}$. Let $M_{R}^{-\infty}(X)$ denote the space of smoothing Mellin symbols with asymptotic type $R$, defined to be the set of all $f(z) \in \mathcal{A}\left(\mathbb{C} \backslash \pi_{\mathbb{C}} R, L^{-\infty}(X)\right)$ that are meromorphic with poles at the points $r_{j}$ of multiplicity $n_{j}+1$ with Laurent coefficients at $\left(z-r_{j}\right)^{-(k+1)}$ being finite rank operators in $L^{-\infty}(X), 0 \leq k \leq n_{j}$, such that for every $\pi_{\mathbb{C}} R$-excision function $\chi(z)$ we have $\left.\chi f\right|_{\Gamma_{\beta}} \in L^{-\infty}\left(X ; \Gamma_{\beta}\right)$ for every $\beta \in \mathbb{R}$, uniformly in compact $\beta$-intervals.

Let us set

$$
M_{R}^{m}(X):=M_{\mathcal{O}}^{m}(X)+M_{R}^{-\infty}(X)
$$


which means the space of all $h+f$ with $h$ in the first, $f$ in the second space on the right hand side. The spaces (1.8) of meromorphic operator functions have many remarkable properties. First it is clear that the $z$-wise composition preserves the spaces, i.e., we have

$$
f \in M_{R}^{m}(X), \tilde{f} \in M_{\tilde{R}}^{\tilde{m}}(X) \Rightarrow f \tilde{f} \in M_{S}^{m+\tilde{m}}(X)
$$

for a resulting asymptotic type $S$.

An element $f \in M_{R}^{m}(X)$ is called elliptic (of order $m$ ) if for some $\beta \in \mathbb{R}$, for $\Gamma_{\beta} \cap \pi_{\mathbb{C}} R=\emptyset$, the family $\left.f\right|_{\Gamma_{\beta}} \in L_{\mathrm{cl}}^{m}\left(X ; \Gamma_{\beta}\right)$ is parameter-dependent elliptic (of order $m$ ). This condition is independent of $\beta$.

Example 1.1. Let $l(z) \in M_{R}^{-\infty}(X)$; then $f(z)=1+l(z)$ is elliptic of order 0. Moreover, there is an $m(z) \in M_{S}^{-\infty}(X)$ for some $S$ such that $(1+l(z))^{-1}=m(z)$.

Theorem 1.2. Let $f \in M_{R}^{m}(X)$ be elliptic; then there is a (unique) $f^{-1} \in M_{S}^{-m}(X)$ for some asymptotic type $S$ such that $f f^{-1}=1$, and $f^{-1} f=1$ in the sense of the multiplications (1.9).

A proof may be found, for instance, in [7, Section 2.2.5.]. To give an idea on the arguments we can apply Theorem 1.4 below for $l=0$. Up to a translation in the complex plane we may assume that $R \cap \Gamma_{0}=\emptyset$; then $\left.f\right|_{\Gamma_{0}}$ has a parameter-dependent parametrix $k \in L_{\mathrm{cl}}^{-m}\left(X ; \Gamma_{0}\right)$. It follows that $V(\psi) k \in M_{\mathcal{O}}^{-\infty}\left(X ; \Gamma_{0}\right)$ and $f V(\psi) k=1+l$ for some $l \in M_{P}^{-\infty}(X)$ for some $P$. Then it suffices to apply the observation of Example 1.1 together with relation (1.9) and to set $f^{-1}=(V(\psi) k)(1+l)^{-1}$.

Let us now sketch some aspects of the cone algebra on a compact manifold $M$ with conical singularities. To illustrate the sitiuation let us first introduce the subspace $L_{\mathrm{deg}}^{m}(M \backslash\{v\})$ of all $A \in L_{\mathrm{cl}}^{m}(M \backslash\{v\})$ that are $\bmod L^{-\infty}(M \backslash\{v\})$ locally close to $v$ of the form $r^{-m} \mathrm{Op}_{r}(a), a(r, \rho)=$ $\tilde{a}(r, r \rho)$ for some $\tilde{a}(r, \tilde{\rho}) \in C^{\infty}\left(\overline{\mathbb{R}}_{+}, L_{\mathrm{cl}}^{m}\left(X ; \mathbb{R}_{\tilde{\rho}}\right)\right)$. The homogeneous principal symbol $\sigma_{0}(A)$ of an operator $A \in L_{\operatorname{deg}}^{m}(M \backslash\{v\})$ is locally near $v$ in the variables $(r, x) \in X^{\wedge}$ of the form $\sigma_{0}(A)(r, x, \rho, \xi)=r^{-m} \tilde{\sigma}_{0}(r, x, r \rho, \xi)$ for a (so-called reduced) symbol $\tilde{\sigma}_{0}(r, x, \tilde{\rho}, \xi)$, smooth up to $r=0$. The cone algebra over a compact manifold $M$ with conical singularity consists of operators of the form

$$
A=\omega r^{-m} \mathrm{op}_{M}^{\gamma-n / 2}(h+l) \omega^{\prime}+A_{\text {int }}+G
$$

for cut-off functions $\omega, \omega^{\prime}, h(r, z) \in C^{\infty}\left(\overline{\mathbb{R}}_{+}, M_{\mathcal{O}}^{m}(X)\right), l(z) \in M_{R}^{-\infty}(X)$ for some $R$, moreover, $A_{\text {int }} \in L_{\mathrm{cl}}^{m}(M \backslash\{v\})$ supported off some neighbourhood of $v$, and a so-called Green operator $G$, defined by the mapping properties

$$
G: \mathcal{H}^{s, \gamma}(M) \rightarrow \mathcal{H}^{\infty, \gamma-m+\varepsilon}(M), G^{*}: \mathcal{H}^{s,-\gamma+m}(M) \rightarrow \mathcal{H}^{\infty,-\gamma+\varepsilon}(M),
$$

for some $\varepsilon>0$ (depending on $G$ ) and all $s \in \mathbb{R}$ where $G^{*}$ means the formal adjoint with respect to the $\mathcal{H}^{0,0}(M)$-scalar product. The first summand on the right of (1.10) refers to the local variables $(r, x) \in X^{\wedge}$ near $v$. The operators of the form (1.10) exhaust the space $L_{\text {deg }}^{m}(M \backslash\{v\}) \bmod$ $L^{-\infty}(M \backslash\{v\})$. From the space $L_{\mathrm{deg}}^{m}(M \backslash\{v\})$ the operators $A$ inherit $\sigma_{0}(A)$, and $\tilde{\sigma}_{0}(A)$. In addition in the cone algebra we have the principal conormal symbol, defined as the operator family

$$
\sigma_{1}(A)(z):=h(0, z)+l(z): H^{s}(X) \rightarrow H^{s-m}(X)
$$

for $s \in \mathbb{R}$. An operator $A$ in the cone calculus is called $\sigma_{0}$-elliptic if $\sigma_{0}(A)$ never vanishes as a function in $C^{\infty}\left(T^{*}(M \backslash\{v\}) \backslash 0\right)$ and if in addition $\tilde{\sigma}_{0}(A)$ is non-zero up to $r=0$. Moreover, $A$ is called $\sigma_{1}$-elliptic if (1.12) consists of isomorphisms for all $z \in \Gamma_{(n+1) / 2-\gamma}$. We also need a few notions from the cone calculus on the infinite stretched cone $X^{\wedge}$. Here we can express everything in the variables $(r, x)$. The properties of the respective operators close to $r=0$ are as before. An extra assumption concerns $A_{\text {int }}+G$, cf. the formula (1.10). On $A_{\text {int }}$ we assume that it belongs to the classical exit calculus for $r \rightarrow \infty$ of weight 0 at $\infty$. Here we refer to the terminology of [8], and we use the notation for the respective principal exit symbols $\sigma_{\mathrm{E}}:=\left(\sigma_{\mathrm{e}}, \sigma_{\psi, \mathrm{e}}\right)$. The conditions on the Green operators $G$ are replaced by mapping properties referring to the spaces

$$
\mathcal{S}^{\gamma}\left(X^{\wedge}\right), \gamma \in \mathbb{R},
$$


defined as projective limits of the spaces $\langle r\rangle^{-N} \mathcal{K}^{N, \gamma}\left(X^{\wedge}\right)$ over $N \in \mathbb{N}$. The condition is

$$
G: \mathcal{K}^{s, \gamma}\left(X^{\wedge}\right) \rightarrow \mathcal{S}^{\gamma-m+\varepsilon}\left(X^{\wedge}\right), G^{*}: \mathcal{K}^{s,-\gamma+m}\left(X^{\wedge}\right) \rightarrow \mathcal{S}^{-\gamma+\varepsilon}\left(X^{\wedge}\right),
$$

for some $\varepsilon>0$ (depending on $G$ ) and all $s \in \mathbb{R}$ where $G^{*}$ means the formal adjoint with respect to the $\mathcal{K}^{0,0}\left(X^{\wedge}\right)$-scalar product. The role of the $\sigma_{1}$-ellipticity (apart from the $\sigma_{0}$-ellipticity) is illustrated by the following theorem.

Theorem 1.3. Let $A$ be an operator in the cone algebra on a compact manifold $M$ with conical singularity, and fix a weight $\gamma \in \mathbb{R}$. Then the following conditions are equivalent:

(i) $A$ is $\left(\sigma_{0}, \sigma_{1}\right)$-elliptic with respect to $\gamma$ (the latter referring to $\left.\sigma_{1}\right)$;

(ii) the operator (1.4) is a Fredholm operator for some $s=s_{0} \in \mathbb{R}$.

Moreover, let $A$ be an operator in the cone algebra on an infinite stretchend cone $X^{\wedge}$ for smooth closed $X$, and fix $\gamma$. Then the following conditions are equivalent:

(iii) $A$ is $\left(\sigma_{0}, \sigma_{1}, \sigma_{\mathrm{E}}\right)$-elliptic with respect to $\gamma$ (the latter referring to $\left.\sigma_{1}\right)$;

(iv) the operator

$$
A: \mathcal{K}^{s, \gamma}\left(X^{\wedge}\right) \rightarrow \mathcal{K}^{s-m, \gamma-m}\left(X^{\wedge}\right)
$$

is a Fredholm operator for some $s=s_{0} \in \mathbb{R}$.

The Fredholm property then always holds for all $s$. Concerning the proof, cf. [7, Sections 2.2.1. and 2.2.3.].

Next we pass to the kernel cut-off theorem. For purposes below we give the formulation for parameter-dependent operator families with parameter $\lambda \in \mathbb{R}^{l}$; for conical singularities the case $l=0$ is sufficient. Let $M_{\mathcal{O}}^{m}\left(X ; \mathbb{R}^{l}\right)$ defined to be the set of all $h(z, \lambda) \in \mathcal{A}\left(\mathbb{C}, L_{\mathrm{cl}}^{m}\left(X ; \mathbb{R}^{l}\right)\right)$ such that $\left.h\right|_{\Gamma_{\beta} \times \mathbb{R}^{l}} \in L_{\mathrm{cl}}^{m}\left(X ; \Gamma_{\beta} \times \mathbb{R}^{l}\right)$ for every $\beta \in \mathbb{R}$, uniformly in finite $\beta$-intervals.

Consider an element $a(z, \lambda) \in L_{\mathrm{cl}}^{m}\left(X ; \Gamma_{0} \times \mathbb{R}^{l}\right)$, and let $\varphi \in C_{0}^{\infty}\left(\mathbb{R}_{+}\right)$. Then the kernel cut-off operator $V(\varphi)$, applied to a Mellin amplitude function $a$ is defined by the expression

$$
V(\varphi) a(i \rho, \lambda):=\int_{0}^{\infty} \theta^{i \rho} \varphi(\theta) k(a)(\theta, \lambda) \theta^{-1} d \theta
$$

where $k(a)(\theta, \lambda):=\int_{-\infty}^{\infty} \theta^{-i \rho} a(i \rho, \lambda) d \rho$.

Theorem 1.4. (i) For every $\varphi \in C_{0}^{\infty}\left(\mathbb{R}_{+}\right)$and $f(w, \lambda) \in L_{\mathrm{cl}}^{m}\left(X ; \Gamma_{0} \times \mathbb{R}^{l}\right)$ we have $V(\varphi) f \in$ $M_{\mathcal{O}}^{m}\left(X ; \mathbb{R}^{l}\right)$.

(ii) Let $\psi \in C_{0}^{\infty}(\mathbb{R})$ be a function that is equal to 1 in a neighbourhood of the origin; then $\left.V(\psi) f\right|_{\Gamma_{0} \times \mathbb{R}^{l}}=\left.f\right|_{\Gamma_{0} \times \mathbb{R}^{l}} \bmod L^{-\infty}\left(X ; \Gamma_{0} \times \mathbb{R}^{l}\right)$. Setting $\psi_{\varepsilon}(\theta):=\psi(\varepsilon \log \theta), \varepsilon>0$, for every $f(w, \lambda) \in L_{\mathrm{cl}}^{m}\left(X ; \Gamma_{0} \times \mathbb{R}^{l}\right)$ we have

$$
\lim _{\varepsilon \rightarrow 0} V\left(\psi_{\varepsilon}\right) f(w, \lambda)=f(w, \lambda) .
$$

This theorem is known from [7], see also [8]. A new proof was given in [?].

\section{$2 \quad$ Ellipticity with Respect to Prescribed Weights}

Theorem 2.1. Let $h \in M_{\mathcal{O}}^{m}(X)$ such that $\left.h\right|_{\Gamma_{\beta}} \in L_{\mathrm{cl}}^{m}\left(X, \Gamma_{\beta}\right)$ is parameter-dependent elliptic for some real $\beta$. Then for every fixed $\gamma \in \mathbb{R}$ there exists an $f \in M_{\mathcal{O}}^{-\infty}(X)$ such that

$$
(h-f)(z): H^{s}(X) \rightarrow H^{s-m}(X)
$$

is a family of isomorphisms for all $z \in \Gamma_{(n+1) / 2-\gamma}$ and every $s \in \mathbb{R}$. 
Proof. The above-mentioned discrete set $D \in \mathbb{C}$ intersects the weight line $\Gamma_{(n+1) / 2-\gamma}$ in at most finitely many points $\left\{p_{1}, \ldots, p_{N}\right\}$ such that $(2.1)$ is invertible for all $z \in \Gamma_{(n+1) / 2-\gamma} \backslash\left\{p_{1}, \ldots, p_{N}\right\}$. Without loss of generality we assume $\gamma=(n+1) / 2$ since a translation parallel to the real axis allows us to change $\gamma$. Moreover, let $N=1$; the proof easily extends to the case of arbitrary $N$ which is left to the reader. In other words, we assume that (2.1) is invertible for all $z \in \Gamma_{0} \backslash\{p\}$ for some $p \in \Gamma_{0}$. Since (2.1) is a family of classical pseudo-differential operators that are Fredholm and of index 0 , there are finite-dimensional subspaces $V, W \subset C^{\infty}(X), \operatorname{dim} V=\operatorname{dim} W=: d$, such that $V=\operatorname{ker} h(p) \subset C^{\infty}(X)$, and $W+\operatorname{im} h(p)=H^{s-m}(X)$ for every $s \in \mathbb{R}$. Then for any isomorphism $k: \mathbb{C}^{d} \rightarrow W$ the row matrix

$$
\left(\begin{array}{lll}
h & k
\end{array}\right):{\underset{\mathbb{C}}{H^{s}}}_{\mathbb{C}^{d}}^{H^{s-m}}(X)
$$

is surjective for all $s$, and we have $\operatorname{ker}(h \quad k)=\operatorname{ker} h \oplus\{0\}=V \oplus\{0\}$. Let $t_{0}: V \rightarrow \mathbb{C}^{d}$ be an isomorphism, and define a continuous operator $t: L^{2}(X) \rightarrow \mathbb{C}^{d}$ by composing $t_{0}$ with the orthogonal projection $L^{2}(X) \rightarrow V$. Then $t$ extends (or restricts) to a continuous mapping $t$ : $H^{s}(X) \rightarrow \mathbb{C}^{d}$, and

$$
\left(\begin{array}{ll}
h & k \\
t & 0
\end{array}\right): \begin{gathered}
H^{s}(X) \\
\mathbb{C}^{d}
\end{gathered} \rightarrow \begin{gathered}
H^{s-m}(X) \\
\mathbb{C}^{d}
\end{gathered}
$$

is an isomorphism. By using the fact that that linear isomorphisms form an open set in the space of linear continuous operators, for fixed $s=s_{1}$ there is a $c_{1}>0$ such that

$$
\left(\begin{array}{ll}
h & k \\
t & c
\end{array}\right): \underset{\mathbb{C}^{d}}{H^{s}(X)} \rightarrow \begin{gathered}
H^{s-m}(X) \\
\mathbb{C}^{d}
\end{gathered}
$$

is an isomorphism for $s=s_{1}, c:=c_{1} \mathrm{id}_{\mathbb{C}^{d}}$. This implies that (2.2) is an isomorphism for all $s \in \mathbb{R}$ since such a block matrix operator is an isomorphism if and only if the first row is surjective, and the second row maps the kernel of the first one isomorphically to $\mathbb{C}^{d}$. However, $\operatorname{ker}(h \quad k)=\operatorname{ker} h=V$ is independent of $s$; therefore, the criterion is fullfilled for all $s$. From (2.2) we now produce an invertible $2 \times 2$ matrix

$$
\left(\begin{array}{cc}
h-k c^{-1} t & 0 \\
0 & c
\end{array}\right)=\left(\begin{array}{cc}
1 & -k c^{-1} \\
0 & 1
\end{array}\right)\left(\begin{array}{ll}
h & k \\
t & c
\end{array}\right)\left(\begin{array}{cc}
1 & 0 \\
-c^{-1} t & 1
\end{array}\right)
$$

with 1 denoting the identity operator in $\mathbb{C}^{d}$. Since all factors are invertible, also

$$
h-k c^{-1} t: H^{s}(X) \rightarrow H^{s-m}(X)
$$

is invertible. Observe that when we replace the operator $k$ in $(2.2)$ by $\delta k$ for any $\delta>0$ then the kernel of the modified first row is isomorphically mapped by the second row to $\mathbb{C}^{d}$, no matter how large $\delta$ is. Thus the whole construction can be repeated with $\delta k$ instead of $k$ but the same second row $\left(\begin{array}{ll}t & c\end{array}\right)$.

Let us now choose a function $\delta(w) \in C_{0}^{\infty}(I)$ for $I=\left\{w \in \Gamma_{0}:|w-p|<b\right\}$ for some $b>0$, where $\delta(p) \neq 0$. Then, setting

$$
h_{\delta}(w):=(h(w) \quad \delta(w) k): \quad \underset{\mathbb{C}^{d}}{H^{s}(X)} \rightarrow H^{s-m}(X),
$$

for every $\varepsilon>0$ there are $b>0$ and $\delta \in C_{0}^{\infty}(I), \delta(p) \neq 0$, with $\left\|h_{\delta}(w)-h_{\delta}(p)\right\|_{\mathcal{L}\left(H^{s}(X) \oplus \mathbb{C}^{d}, H^{s-m}(X)\right)}<$ $\varepsilon$ for all $w \in I$.

In fact, for sufficiently small $b$ we have $\|h(p)-h(w)\|_{\mathcal{L}\left(H^{s}(X), H^{s-m}(X)\right)}<\varepsilon / 2$ for all $w \in I$, since $h(w)$ is continuous with values in $\mathcal{L}\left(H^{s}(X), H^{s-m}(X)\right)$. Moreover,

$$
\sup _{w \in I}\|\delta(p) k-\delta(w) k\|_{\mathcal{L}\left(\mathbb{C}^{d}, H^{s-m}(X)\right)} \leq \sup _{w \in I}|\delta(p)-\delta(w)|\|k\|_{\mathcal{L}\left(\mathbb{C}^{d}, H^{s}(X)\right)}<\varepsilon / 2
$$


when we choose first $\delta(w) \in C_{0}^{\infty}(I)$ arbitrary, $\delta(p)>0$, and then multiply $\delta$ by a sufficiently small constant $>0$ and denote the new $\delta$ again by $\delta$. Thus

$$
\left(\begin{array}{cc}
h(w) & \delta(w) k \\
t & c
\end{array}\right): \underset{\mathbb{C}^{d}}{H^{s}(X)} \rightarrow \stackrel{H^{s-m}(X)}{\oplus} \underset{\mathbb{C}^{d}}{\oplus}
$$

is a family of isomorphisms for all $w \in I$ for sufficiently small $\epsilon>0$. Analogously as before we see that

$$
h(w)-\delta(w) k c^{-1} t: H^{s}(X) \rightarrow H^{s-m}(X)
$$

is a family of isomorphisms for all $w \in \Gamma_{0}$, first for $w \in I$, but then, since $h(w)$ consists of isomorphisms for $w \neq p$ and $\delta \in C_{0}^{\infty}(I)$, also for $w \notin I$.

In this consideration we have assumed that $s \in \mathbb{R}$ is fixed. However, the left hand side of (2.3) consists of a family of elliptic pseudo-differential operators on $X$; therefore, kernel and cokernel are independent of $s$, and hence we have isomorphisms (2.3) for all $w \in \Gamma_{0}, s \in \mathbb{R}$.

Let us interpret $f_{1}(w):=\delta(w) k c^{-1} t$ as an operator-valued Mellin symbol in the covariable $w \in \Gamma_{0}$, with compact support in $w$ and values in operators $\in L^{-\infty}(X)$ of finite rank.

In a final step of the proof we modify $f_{1}(w)$ to obtain an element $f(w) \in M_{\mathcal{O}}^{-\infty}(X)$ that approximates $f_{1}(w)$ in such a way that (2.1) are isomorphisms for all $w \in \Gamma_{0}$. First Theorem 1.4 gives us $f_{(\varepsilon)}(w):=V\left(\psi_{\varepsilon}\right) f_{1}(w) \in M_{\mathcal{O}}^{-\infty}(X)$, and $f_{(\varepsilon)} \rightarrow f_{1}$ as $\varepsilon \rightarrow 0$ in the topology of $L^{-\infty}\left(X ; \Gamma_{0}\right)$. We will show that we may set $f(w)=f_{(\varepsilon)}(w)$ for any fixed sufficiently small $\varepsilon>0$. It is evident that for any fixed compact interval $K \subset \Gamma_{0}$ containing the point $p$ there is an $\varepsilon(K)>0$ such that for all $0<\varepsilon<\varepsilon(K)$ we have isomorphisms (2.1) for all $w \in K$. To argue for $w \in \Gamma_{0} \backslash K$ we employ Theorem 1.2, i.e., there is an element $h^{-1}(w) \in M_{S}^{-m}(X)$ for some discrete Mellin asymptotic type $S$ with $\pi_{\mathbb{C}} S \cap \Gamma_{0}=\{p\}$ such that $h^{-1}(w) h(w)=1$. This gives us family of continuous operators

$$
1-h^{-1}(w) f_{(\varepsilon)}(w): H^{s}(X) \rightarrow H^{s}(X)
$$

parametrised by $w \in \Gamma_{0} \backslash K$. Without loss of generality we take $K$ so large that supp $f_{1} \subseteq K_{1}$ for some subinterval $K_{1} \subset \operatorname{int} K$. Let $\chi(w) \in C^{\infty}\left(\Gamma_{0}\right)$ be any function which is equal to 0 in a neighbourhood of supp $f_{1}$ and 1 outside an open $U \subset \Gamma_{0}, K_{1} \subset U$ with $\bar{U} \subset K$. Then $f_{(\varepsilon)} \rightarrow f_{1}$ gives us $\chi f_{(\varepsilon)} \rightarrow 0$ as $\varepsilon \rightarrow 0$ in the space $\mathcal{S}\left(\Gamma_{0}, L^{-\infty}(X)\right)$. Using that $h^{-1}(w)$ is a parameter-dependent family of operators in $L_{\mathrm{cl}}^{-m}(X)$ with parameter $w \in \Gamma_{0} \backslash\{p\}$ we also obtain $h^{-1} \chi f_{(\varepsilon)} \rightarrow 0$ in $\mathcal{S}\left(\Gamma_{0}, L^{-\infty}(X)\right)$. It follows that there is an $\tilde{\varepsilon}(K)>0$ such that (2.4) are isomorphisms for all $0<\varepsilon<\tilde{\varepsilon}(K)$ and all $w \in \Gamma_{0} \backslash K$. Thus we obtain altogether isomorphisms (2.1) for $f(w)=$ $f_{(\varepsilon)}(w), 0<\varepsilon<\min (\varepsilon(K), \tilde{\varepsilon}(K))$ for all $w \in \Gamma_{0}$.

Corollary 2.2. Let $h \in M_{R}^{m}(X)$ be elliptic. Then for every $\gamma \in \mathbb{R}$ there exists an $f \in M_{R}^{-\infty}(X)$ such that (2.1) is a family of isomorphisms for all $z \in \Gamma_{(n+1) / 2-\gamma}$ and every $s \in \mathbb{R}$.

Proof. According to (1.8) we have a decomposition $h=h_{0}+f_{0}$ for $h_{0} \in M_{\mathcal{O}}^{m}(X), f_{0} \in M_{R}^{-\infty}(X)$. From Theorem 2.1 we find an $f_{1} \in M_{\mathcal{O}}^{-\infty}(X)$ such that $\left(h_{0}-f_{1}\right)(z)=\left(h-f_{0}-f_{1}\right)(z): H^{s}(X) \rightarrow$ $H^{s-m}(X)$ is a family of isomorphisms for all $z \in \Gamma_{(n+1) / 2-\gamma}$ and every $s \in \mathbb{R}$. Thus it suffices to set $f=f_{0}+f_{1}$.

Theorem 2.1 and Corollary 2.2 can be applied to the construction of parametrices in the cone algebra. Although it has been known for a long time that the cone algebra is closed under forming parametrices of elliptic elements, cf. [?], [7], it is desirable to formally apply the following simple guideline. Assume we are given an operator algebra with a principal symbolic hierarchy $\sigma(A)=$ $\left(\sigma_{j}(A)\right)_{0 \leq j \leq k}$ where $\sigma$-ellipticity means a bijectivity condition for every $\sigma_{j}(A)$, and $\sigma$-ellipticity of two operators implies the same for the composition. Moreover, assume that there are operator conventions that produce operators for a given symbol. If for an operator $B$ which is elliptic with respect to the symbolic components $\sigma_{j}, 0 \leq j \leq l$ for an $l<k$ we always find a $\sigma$-elliptic operator $Q$ such that $\sigma_{j}(Q)=\sigma_{j}(B)$ for all $j=0, \ldots, l$, then the process is as follows. Assume $A=: A_{0}$ is $\sigma$-elliptic. Then we consider $\sigma_{0}\left(A_{0}\right)$, form an operator $B_{0}$ such that $\sigma_{0}\left(B_{0}\right)=\sigma_{0}\left(A_{0}\right)^{-1}$ and pass 
to a $\sigma$-elliptic $P_{0}$ with $\sigma_{0}\left(P_{0}\right)=\sigma_{0}\left(B_{0}\right)$. Then the operator $A_{1}:=P_{0} A_{0}$ is $\sigma$-elliptic and has the property $\sigma_{0}\left(A_{1}\right)=1$. The next step is to form a $B_{1}$ with $\sigma_{j}\left(B_{1}\right)=\sigma_{j}\left(A_{1}\right)^{-1}$ for $j=0,1$ and pass to a $\sigma$-elliptic $P_{1}$ with $\sigma_{j}\left(P_{1}\right)=\sigma_{j}\left(B_{1}\right)$ for $j=0,1$. Then the operator $A_{2}:=P_{1} A_{1}=P_{1} P_{0} A_{0}$ is $\sigma$-elliptic and has the property $\sigma_{j}\left(A_{2}\right)=1$ for $j=0,1$. By continuing this process we find a $\sigma$-elliptic $P_{k}$ such that $P:=P_{k} P_{k-1} \cdots P_{0}$ has the property $\sigma_{j}(P A)=1$ or $\sigma_{j}(P)=\sigma_{j}(A)^{-1}$ for $j=0, \ldots k$. If the algebra admits a formal Neumann series argument (which is in our application the case) then from $P$ we obtain a parametrix of $A$ with smoothing left-over terms.

Here for the cone algebra we have $k=1$, and the above-mentioned step from $B$ to $Q$ is just what we can do by Theorem 2.1 and Corollary 2.2.

\section{The Case of Manifolds with Conical Singularities and Boundary}

Let $M$ be a manifold with conical singularities and boundary. For simplicity we assume again that there is one conical point $v$. Then $M \backslash\{v\}$ is a $C^{\infty}$ manifold with boundary. Moreover, $M$ is locally near $v$ modelled on a cone $X^{\Delta}$ where $X$ is a compact $C^{\infty}$ manifold with boundary, similarly as at the beginning of Section 1 . Let $2 M$ and $2 X$ be the doubles of $M$ and $X$, respectively (defined as $C^{\infty}$ manifolds obtained by gluing together two copies of the respective manifolds along the common boundary). Then $2 M$ is a manifold with conical point as in Section 1 and $2 X$ is the base of the local cone.

On the non-compact smooth manifold $M \backslash\{v\}$ with boundary we have the pseudo-differential calculus of boundary value problems (BVPs) with the transmission property at the boundary, cf. [2], consisting of spaces $\mathcal{B}^{m, d}(M \backslash\{v\}), m \in \mathbb{Z}, d \in \mathbb{N}$, of order $m$ and type $d$. The BVPs themselves are represented by $2 \times 2$ block matrices of operators

$$
A: C_{0}^{\infty}\left(\operatorname{int}(M \backslash\{v\}), E_{1}\right) \oplus C_{0}^{\infty}\left(\partial(M \backslash\{v\}), J_{1}\right) \rightarrow C^{\infty}\left(\operatorname{int}(M \backslash\{v\}), E_{2}\right) \oplus C^{\infty}\left(\partial(M \backslash\{v\}), J_{2}\right)
$$

for smooth complex vector bundles $E_{k}$ over $M \backslash\{v\}$ and $J_{k}$ over $\partial(M \backslash\{v\})$, respectively, $k=1,2$. Writing $A=\left(A_{i j}\right)_{i, j=1,2}$ we have $A_{11}=\mathrm{r}^{+} A \mathrm{e}^{+}+G$ for $A \in L_{\mathrm{tr}}^{m}\left(2 M \backslash\{v\}, 2 E_{1}, 2 E_{2}\right)$ which is the space of classical pseudo-differential operators over the respective manifold with the transmission property at the boundary, acting between distributional sections of corresponding extensions of the indicated vector bundles to $2 M \backslash\{v\}$, and $\mathrm{e}^{+}, \mathrm{r}^{+}$denote extension by zero to the opposite side of the double and restriction to int $(M \backslash\{v\})$. Moreover, $G$ is a so-called Green operator of order $m$ and type $d$ (the latter means, roughly speaking, an involved differentiation of order $d$ transversally to the boundary), while $A_{21}$ is a trace operator of order $m+1 / 2$ and type $d$ (representing boundary conditions), $K$ is a potential operator of order $m-1 / 2$, and $Q \in L_{\mathrm{cl}}^{m}\left(\partial(M \backslash\{v\}) ; J_{1}, J_{2}\right)$.

In the following we suppress the bundles $E_{k}, J_{k}$ in the notation and formally assume that those are trivial and of fibre dimension 1. All considerations are, of course, valid in general. The operators $A \in \mathcal{B}^{m, d}$ over a smooth manifold with boundary have a principal symbolic structure $\left(\sigma_{0}(A), \sigma_{0}^{\prime}(A)\right)$ where $\sigma_{0}(A)$ is the standard homogeneous principal symbol of $A$ of order $m$ and $\sigma_{0}^{\prime}(A)$ the (operator-valued) homogeneous principal boundary symbol of $A$. If $A \in \mathcal{B}^{m, d}, \tilde{A} \in \mathcal{B}^{\tilde{m}, \tilde{d}}$, and $A$ or $\tilde{A}$ properly supported, we have $A \tilde{A} \in \mathcal{B}^{m+\tilde{m}, \tilde{d}}$ for $\tilde{\tilde{d}}=\max \{\tilde{m}+d, \tilde{d}\}$, and $\sigma_{0}(A \tilde{A})=$ $\sigma_{0}(A) \sigma_{0}(\tilde{A}), \sigma_{0}^{\prime}(A \tilde{A})=\sigma_{0}^{\prime}(A) \sigma_{0}^{\prime}(\tilde{A})$.

We do not recall here all details on the cone calculus of BVPs; more details may be found in [?]. What we do here is to answer the question of conormal ellipticity for a prescribed weight, and we formulate the tools up to the point where it becomes clear how the analogue of the approach of Section 2 works. The general frame of the cone calculus of BVPs is the subspace $\mathcal{B}_{\mathrm{deg}}^{m, d}(M \backslash\{v\})$ of degenerate operators in $\mathcal{B}^{m, d}(M \backslash\{v\})$ that are locally near $v$ defined in terms of $\mathcal{B}^{m, d}(X)$-valued symbols, more precisely, where the covariables cotain a parameter $\lambda \in \mathbb{R}^{l}$ from a parameter-dependent analogue $\mathcal{B}^{m, d}\left(X ; \mathbb{R}^{l}\right)$ of $\mathcal{B}^{m, d}(X)$ (here for $\left.l=1\right)$. Using the fact that $\mathcal{B}^{m, d}(X ; \mathbb{R})$ is a Fréchet space in a natural way we can form operator functions $\tilde{f}(r, \tilde{\rho}) \in C^{\infty}\left(\overline{\mathbb{R}}_{+}, \mathcal{B}^{m, d}\left(X ; \mathbb{R}_{\tilde{\rho}}\right)\right)$. Then $\mathcal{B}_{\mathrm{deg}}^{m, d}(M \backslash\{v\})$ is defined as the subspace of all $A \in \mathcal{B}^{m, d}(M \backslash\{v\})$ that are $\bmod \mathcal{B}^{-\infty, d}(M \backslash\{v\})$ locally near $v$ 
in the splitting of variables $(r, x) \in \mathbb{R}_{+} \times X$ of the form $r^{-m} \mathrm{Op}_{r}(f), f(r, \rho)=\tilde{f}(r, r \rho)$, for some $\tilde{f}(r, \tilde{\rho})$ of the above-mentioned kind. Apart from the pair of principal symbols $\sigma_{0}(A), \sigma_{0}^{\prime}(A)$ of $A$ interpreted as an element of $\mathcal{B}^{m, d}(M \backslash\{v\})$ for $A \in \mathcal{B}_{\mathrm{deg}}^{m, d}(M \backslash\{v\})$ we have the pair of reduced symbols $\tilde{\sigma}_{0}(A), \tilde{\sigma}_{0}^{\prime}(A)$ in the variables $(r, x) \in X^{\wedge}$ and $\left(r, x^{\prime}\right) \in \partial X^{\wedge}$, respectively, defined by $\tilde{\sigma}_{0}(A)(r, x, \rho, \xi)=r^{m} \sigma_{0}(A)\left(r, x, r^{-1} \rho, \xi\right)$ and $\tilde{\sigma}_{0}^{\prime}(A)\left(r, x^{\prime}, \rho, \xi^{\prime}\right)=r^{m} \sigma_{0}^{\prime}(A)\left(r, x^{\prime}, r^{-1} \rho, \xi^{\prime}\right)$ where $\tilde{\sigma}_{0}(A), \tilde{\sigma}_{0}^{\prime}(A)$ are smooth up to $r=0$.

Let $\mathcal{M}_{\mathcal{O}}^{m, d}(X)$ defined to be the space of all $h \in \mathcal{A}\left(\mathbb{C}, \mathcal{B}^{m, d}(X)\right)$ such that $\left.h\right|_{\Gamma_{\beta}} \in \mathcal{B}^{m, d}\left(X ; \Gamma_{\beta}\right)$ for every $\beta \in \mathbb{R}$, uniformly in compact $\beta$-intervals. Moreover, for a sequence (1.7) we define the space $\mathcal{M}_{R}^{-\infty, d}(X)$ as the set of all $f(z) \in \mathcal{A}\left(\mathbb{C} \backslash R, \mathcal{B}^{-\infty, d}(X)\right)$ which are meromorphic with poles at the points $r_{j}$ of multiplicity $n_{j}+1$, where the Laurent coefficients at $\left(z-r_{j}\right)^{-(k+1)}$ are finite rank operators in $\mathcal{B}^{-\infty, d}(X)$ for $0 \leq k \leq n_{j}$, and such that for every $R$-excision function $\chi(z)$ we have $\left.\chi f\right|_{\Gamma_{\beta}} \in \mathcal{B}^{-\infty, d}\left(X ; \Gamma_{\beta}\right)$ for every $\beta \in \mathbb{R}$, uniformly in compact $\beta$-intervals. Then we set

$$
\mathcal{M}_{R}^{m, d}(X):=\mathcal{M}_{\mathcal{O}}^{m, d}(X)+\mathcal{M}_{R}^{-\infty, d}(X)
$$

An element $f \in \mathcal{M}_{R}^{m, d}(X)$ is called elliptic if for some $\beta \in \mathbb{R}$ for $\Gamma_{\beta} \cap R=\emptyset$ the family $f \mid \Gamma_{\beta} \in$ $\mathcal{B}^{m, d}\left(X ; \Gamma_{\beta}\right)$ is parameter-dependent elliptic. This property is again independent of $\beta$.

Theorem 3.1. Let $f \in \mathcal{M}_{R}^{m, d}(X)$ be elliptic; then there is a (unique) $f^{-1} \in \mathcal{M}_{S}^{-m,(d-\mu)^{+}}(X), \nu^{+}:=$ $\max \{\nu, 0\}$, for some $S$ such that $f f^{-1}=1$ and $f^{-1} f=1$.

The proof of Theorem 3.1 may be obtained by applying similar arguments as for Theorem 1.2. The cone algebra over a compact manifold $M$ with conical singularity $v$ and boundary consisits of operators of the form

$$
A=\omega r^{-m} \mathrm{op}_{M}^{\gamma-n / 2}(h+l) \omega^{\prime}+A_{\mathrm{int}}+G
$$

for cut-off functions $\omega, \omega^{\prime}, h(r, z) \in C^{\infty}\left(\overline{\mathbb{R}}_{+}, \mathcal{M}_{\mathcal{O}}^{m, d}(X)\right), l(z) \in \mathcal{M}_{R}^{-\infty, d}(X)$ for some $R$, moreover, $A_{\text {int }} \in \mathcal{B}^{m, d}(M \backslash\{v\})$ supported off some neighbourhood of $v$, and a so-called Green operator $G$. For type $d=0$ such a $G$ is defined by the mapping properties

$$
G: \mathcal{H}^{s, \gamma}(M) \oplus \mathcal{H}^{s-1 / 2, \gamma-1 / 2}(\partial M) \rightarrow \mathcal{H}^{\infty, \gamma-m+\varepsilon}(M) \oplus \mathcal{H}^{\infty, \gamma-m-1 / 2+\varepsilon}(\partial M),
$$

and, similarly, for the formal adjoint, for some $\varepsilon>0$ (depending on $G$ ) and all $s>1 / 2$. Moreover, a Green operator of the cone calculus of type $d \in \mathbb{N}$ has the form $\sum_{j=0}^{d} G_{j} \operatorname{diag}\left(1, \partial_{t}^{j}\right)$ for Green operators of $G_{j}$ of type 0 where $\partial_{t}^{j}$ is an abbreviation of a $j$-th order differential operator which is close to the boundary differentiating in $t$, the normal direction. The first summand on the right of (1.10) refers to the local variables $(r, x) \in X^{\wedge}$ near $v$. The operators of the form (3.3) exhaust the space $\mathcal{B}_{\mathrm{deg}}^{m, d}(M \backslash\{v\}) \bmod \mathcal{B}^{-\infty, d}(M \backslash\{v\})$. From the space $\mathcal{B}_{\mathrm{deg}}^{m, d}(M \backslash\{v\})$ the operators $A$ inherit the symbols $\sigma_{0}(A), \sigma_{0}^{\prime}(A)$ and $\tilde{\sigma}_{0}(A), \tilde{\sigma}_{0}^{\prime}(A)$. By $\left(\sigma_{0}, \sigma_{0}^{\prime}\right)$-ellipticity of $A$ we understand the condition of non-vanishing of $\sigma_{0}(A)$ and bijectivity of $\sigma_{0}^{\prime}(A)$ (including the case with tilde, then up to $r=0$ ). In addition in the cone algebra we have the principal conormal symbol, defined as the operator family

$$
\sigma_{1}(A)(z):=h(0, z)+l(z): H^{s}(X) \oplus H^{s-1 / 2}(\partial X) \rightarrow H^{s-m}(X) \oplus H^{s-m-1 / 2}(\partial X)
$$

for $s \in \mathbb{R}, s>\max \{m, d\}-1 / 2$. Moreover, $A$ is called $\sigma_{1}$-elliptic if (3.5) consists of isomorphisms for all $z \in \Gamma_{(n+1) / 2-\gamma}$. The operators $A$ in the cone algebra of BVPs on a compact manifold $M$ with conical singularities and boundary induce continuous operators

$$
A: \mathcal{H}^{s, \gamma}(M) \oplus \mathcal{H}^{s-1 / 2, \gamma-1 / 2}(\partial M) \rightarrow \mathcal{H}^{s-m, \gamma-m}(M) \oplus \mathcal{H}^{s-m-1 / 2, \gamma-m-1 / 2}(\partial M)
$$

for all $s \in \mathbb{R}, s>\max \{m, d\}-1 / 2$.

Let us also recall a few notions from the cone calculus on the infinite stretched cone $X^{\wedge}$. Again we can express everything in the variables $(r, x)$. The properties of the respective operators close to $r=0$ are as before. An extra assumption concerns $A_{\text {int }}+G$, cf. the formula (3.3). On $A_{\text {int }}$ 
we assume that it belongs to the classical exit calculus for $r \rightarrow \infty$ of weight 0 at $\infty$. Here we refer to the terminology of [4], and we use the notation for the respective principal exit symbols $\sigma_{\mathrm{E}}:=\left(\sigma_{\mathrm{e}}, \sigma_{\psi, \mathrm{e}}, \sigma_{\mathrm{e}}, \sigma_{\partial, \mathrm{e}}\right)$. The conditions on the Green operators $G$ are replaced by mapping properties referring to the spaces

$$
\mathcal{K}^{s, \gamma}\left(X^{\wedge}\right)=\left.\mathcal{K}^{s, \gamma}\left(2 X^{\wedge}\right)\right|_{\operatorname{int} X^{\wedge}}, \quad \mathcal{S}^{\gamma}\left(X^{\wedge}\right)=\left.\mathcal{S}^{\gamma}\left(2 X^{\wedge}\right)\right|_{X^{\wedge}}, \quad \gamma \in \mathbb{R}
$$

The condition for $d=0$ is

$$
G: \mathcal{K}^{s, \gamma}\left(X^{\wedge}\right) \oplus \mathcal{K}^{s-1 / 2, \gamma-1 / 2}\left(\partial X^{\wedge}\right) \rightarrow \mathcal{S}^{\gamma-m+\varepsilon}\left(X^{\wedge}\right) \oplus \mathcal{S}^{\gamma-m+\varepsilon}\left(\partial X^{\wedge}\right)
$$

for some $\varepsilon>0$ (depending on $G$ ) and all $s \in \mathbb{R}, s>1 / 2$ and an analogous condition for the formal adjoint. In the case $d \in \mathbb{N}, d>0$ the definition of Green operators is similar as before, with differentiations up to order $d$, transversal to the boundary.

Theorem 3.2. Let $A$ be an operator in the cone algebra of BVPs on the compact manifold with conical singularity $M$ with boundary, and fix a weight $\gamma \in \mathbb{R}$. Then the following conditions are equivalent:

(i) $A$ is $\left(\sigma_{0}, \sigma_{0}^{\prime}, \sigma_{1}\right)$-elliptic with respect to $\gamma$ (the latter referring to $\left.\sigma_{1}\right)$;

(ii) the operator (3.6) is a Fredholm operator for some $s=s_{0} \in \mathbb{R}$.

Moreover, let $A$ be an operator in the cone algebra $\mathcal{B}^{m, d}\left(X^{\wedge}\right)$ on an infinite stretched cone $X^{\wedge}$ for a smooth compact manifold $X$ with boundary, and fix $\gamma$. Then the following conditions are equivalent:

(iii) $A$ is $\left(\sigma_{0}, \sigma_{0}^{\prime}, \sigma_{1}, \sigma_{\mathrm{E}}\right)$-elliptic with respect to $\gamma$ (the latter referring to $\left.\sigma_{1}\right)$;

(iv) the operator

$$
A: \mathcal{K}^{s, \gamma}\left(X^{\wedge}\right) \oplus \mathcal{K}^{s, \gamma}\left(\partial X^{\wedge}\right) \rightarrow \mathcal{K}^{s-m, \gamma-m}\left(X^{\wedge}\right) \oplus \mathcal{K}^{s-m, \gamma-m}\left(\partial X^{\wedge}\right)
$$

is a Fredholm operator for some $s=s_{0} \in \mathbb{R}, s_{0}>\max \{m, d\}-1 / 2$.

The Fredholm property then always holds for all $s>\max \{m, d\}-1 / 2$. The tools for the proof of Theorem 3.2 are of a completely analogous structure as those for Theorem 1.3. Let us now turn to an analogue of the kernel cut-off theorem. Let us again give the formulation for parameterdependent operator families with parameter $\lambda \in \mathbb{R}^{l}$; for conical singularities the case $l=0$ is sufficient. Let $\mathcal{M}_{\mathcal{O}}^{m, d}\left(X ; \mathbb{R}^{l}\right)$ defined to be the set of all $h(z, \lambda) \in \mathcal{A}\left(\mathbb{C}, \mathcal{B}^{m, d}\left(X ; \mathbb{R}^{l}\right)\right)$ such that $\left.h\right|_{\Gamma_{\beta} \times \mathbb{R}^{l}} \in \mathcal{B}^{m, d}\left(X ; \Gamma_{\beta} \times \mathbb{R}^{l}\right)$ for every $\beta \in \mathbb{R}$, uniformly in finite $\beta$-intervals.

Consider an element $a(z, \lambda) \in \mathcal{B}^{m, d}\left(X ; \Gamma_{0} \times \mathbb{R}^{l}\right)$, and let $\varphi \in C_{0}^{\infty}\left(\mathbb{R}_{+}\right)$. Then the kernel cut-off operator $V(\varphi)$, applied to a Mellin amplitude function $a$ is defined by the expression

$$
V(\varphi) a(i \rho, \lambda):=\int_{0}^{\infty} \theta^{i \rho} \varphi(\theta) k(a)(\theta, \lambda) \theta^{-1} d \theta
$$

where $k(a)(\theta, \lambda):=\int_{-\infty}^{\infty} \theta^{-i \rho} a(i \rho, \lambda) d \rho$.

Theorem 3.3. (i) For every $\varphi \in C_{0}^{\infty}\left(\mathbb{R}_{+}\right)$and $f(w, \lambda) \in \mathcal{B}^{m, d}\left(X ; \Gamma_{0} \times \mathbb{R}^{l}\right)$ we have $V(\varphi) f \in$ $\mathcal{M}_{\mathcal{O}}^{m, d}\left(X ; \mathbb{R}^{l}\right)$.

(ii) Let $\psi \in C_{0}^{\infty}(\mathbb{R})$ be a function that is equal to 1 in a neighbourhood of the origin; then $\left.V(\psi) f\right|_{\Gamma_{0} \times \mathbb{R}^{l}}=\left.f\right|_{\Gamma_{0} \times \mathbb{R}^{l}} \bmod \mathcal{B}^{-\infty, d}\left(X ; \Gamma_{0} \times \mathbb{R}^{l}\right)$. Setting $\psi_{\varepsilon}(\theta):=\psi(\varepsilon \log \theta), \varepsilon>0$, for every $f(w, \lambda) \in \mathcal{B}^{m, d}\left(X ; \Gamma_{0} \times \mathbb{R}^{l}\right)$ we have

$$
\lim _{\varepsilon \rightarrow 0} V\left(\psi_{\varepsilon}\right) f(w, \lambda)=f(w, \lambda) .
$$


Theorem 3.4. Let $h \in \mathcal{M}_{\mathcal{O}}^{m, d}(X)$ such that $\left.h\right|_{\Gamma_{\beta}} \in \mathcal{B}^{m, d}\left(X, \Gamma_{\beta}\right)$ is parameter-dependent elliptic for some real $\beta$. Then for every fixed $\gamma \in \mathbb{R}$ there exists an $f \in \mathcal{M}_{\mathcal{O}}^{-\infty, d}(X)$ such that

$$
(h-f)(z): H^{s}(X) \oplus H^{s-1 / 2}(\partial X) \rightarrow H^{s-m}(X) \oplus H^{s-m-1 / 2}(\partial X)
$$

is a family of isomorphisms for all $z \in \Gamma_{(n+1) / 2-\gamma}$ and every $s \in \mathbb{R}$.

Proof. An inspection of the proof of Theorem 2.1 shows that the tools are available in analogous form in the case of BVPs. This mainly concerns Theorem 3.1 and Theorem 3.3 . So we can proceed in an analogous manner as in the case of closed manifolds.

Corollary 3.5. Let $h \in \mathcal{M}_{R}^{m, d}(X)$ be elliptic. Then for every $\gamma \in \mathbb{R}$ there exists an $f \in \mathcal{M}_{R}^{-\infty, d}(X)$ such that (3.11) is a family of isomorphisms for all $z \in \Gamma_{(n+1) / 2-\gamma}$ and every $s \in \mathbb{R}$.

Proof. The arguments are analogous to those for Corollary 2.2.

\section{Some Consequences for Edge Symbols}

Let us draw some conclusions of Theorems 2.1 and 3.4 to symbols of the edge calculus. First, a manifold $M$ with edge $Y:=s_{1}(M)$ is characterised by the conditons that $M \backslash Y$ is smooth, and $M$ is locally near $Y$ modelled on wedges $X^{\Delta} \times \Omega$, for a (here) compact smooth manifold $X$ (without/with boundary) and open $\Omega \subseteq \mathbb{R}^{q}$ corresponding to a chart on $Y, q=\operatorname{dim} Y$. The typical differential operators of the edge calculus have locally near $Y$ in the splitting of variables $(r, x, y) \in \mathbb{R}_{+} \times X \times \Omega$ the form

$$
A=r^{-m} \sum_{j+|\alpha| \leq m} a_{j \alpha}(r, y)\left(-r \partial_{r}\right)^{j}\left(r D_{y}\right)^{\alpha}
$$

for coefficients $a_{j \alpha}(r, y) \in C^{\infty}\left(\overline{\mathbb{R}}_{+} \times \Omega\right.$, Diff $\left.{ }^{m-j-|\alpha|}(X)\right)$. In the following discussion we first consider the case $\partial X=\emptyset$. Then the principal symbolic structure of $A$ consists of a pair $\sigma(A):=$ $\left(\sigma_{0}(A), \sigma_{1}(A)\right)$ with $\sigma_{0}(A)$ being the standard homogeneous principal symbol as a function on $T^{*}(M \backslash Y) \backslash 0$, accompanied by the reduced symbol $\tilde{\sigma}_{0}(A)$ defined in the variables $(r, x, y) \in$ $\mathbb{R}_{+} \times X \times \Omega$ by the relation $\tilde{\sigma}_{0}(A)(r, x, y, \rho, \xi, \eta)=r^{m} \sigma_{0}(A)\left(r, x, y, r^{-1} \rho, \xi, r^{-1} \eta\right)$. Moreover, the homogeneous principal edge symbol of $A$

$$
\sigma_{1}(A)(y, \eta)=r^{-m} \sum_{j+|\alpha| \leq m} a_{j \alpha}(0, y)\left(-r \partial_{r}\right)^{j}(r \eta)^{\alpha},
$$

is interpreted as a family of operators

$$
\sigma_{1}(A)(y, \eta)=\mathcal{K}^{s, \gamma}\left(X^{\wedge}\right) \rightarrow \mathcal{K}^{s-m, \gamma-m}\left(X^{\wedge}\right),
$$

on the open stretched cone $X^{\wedge}$, parametrised by $(y, \eta) \in \Omega \times\left(\mathbb{R}^{q} \backslash\{0\}\right)$. Pseudo-differential edge symbols in general are associated with operator-valued amplitude functions of the form

$$
a(y, \eta)=r^{-m} \operatorname{oop}_{M}^{\gamma-n / 2}(h)(y, \eta) \epsilon^{\prime}+(m+g)(y, \eta)
$$

for cut-off functions $\epsilon, \epsilon^{\prime}$, functions $h(r, y, z, \eta)=\tilde{h}(r, y, z, r \eta), \tilde{h}(r, y, z, \tilde{\eta}) \in C^{\infty}\left(\overline{\mathbb{R}}_{+} \times \Omega, M_{\mathcal{O}}^{m}\left(X ; \mathbb{R}^{q}\right)\right)$, cf. the notation in connection with Theorem 1.4 (i), and so-called smoothing Mellin plus Green symbols of order $m$. Those are specific classical operator-valued symbols. The general definition is as follows.

We say that a Hilbert space $H$ is equipped with a group action $\kappa=\left\{\kappa_{\lambda}\right\}_{\lambda \in \mathbb{R}_{+}}$if $\kappa_{\lambda}: H \rightarrow H$ is an isomorphism for every $\lambda \in \mathbb{R}_{+}, \kappa_{\lambda} \kappa_{\lambda^{\prime}}=\kappa_{\lambda \lambda^{\prime}}$ for every $\lambda, \lambda^{\prime} \in \mathbb{R}_{+}$, and if the group is strongly continuous. For two Hilbert spaces $H$ and $\tilde{H}$ with group action $\kappa$ and $\tilde{\kappa}$, respectively, 
the symbol space $S^{m}\left(U \times \mathbb{R}^{q} ; H, \tilde{H}\right)$ for $U \subseteq \mathbb{R}^{d}$ open, $m \in \mathbb{R}$, is defined as the set of all $a(y, \eta) \in C^{\infty}\left(U \times \mathbb{R}^{q}, \mathcal{L}(H, \tilde{H})\right)$ such that

$$
\left\|\tilde{\kappa}_{\langle\eta\rangle}^{-1}\left\{D_{y}^{\alpha} D_{\eta}^{\beta} a(y, \eta)\right\} \kappa_{\langle\eta\rangle}\right\|_{\mathcal{L}(H, \tilde{H})} \leq c\langle\eta\rangle^{m-|\beta|}
$$

for all $(y, \eta) \in K \times \mathbb{R}^{q}, K \subset \subset U$, and $\alpha \in \mathbb{N}^{d}, \beta \in \mathbb{N}^{q}$, for constants $c=c(\alpha, \beta, K)>0$. Moreover, the subspace of classical symbols $S_{\mathrm{cl}}^{m}\left(U \times \mathbb{R}^{q} ; H, \tilde{H}\right)$ is defined by asymptotic expansions of terms $\chi(\eta) a_{(m-j)}(y, \eta)$ for any excision function $\chi$ and homogeneous components $a_{(m-j)}(y, \eta), j \in$ $\mathbb{N},(y, \eta) \in U \times\left(\mathbb{R}^{q} \backslash\{0\}\right) ;$ homogeneity means $a_{(m-j)}(y, \lambda \eta)=\lambda^{m-j} \tilde{\kappa}_{\lambda} a_{(m-j)}(y, \eta) \kappa_{\lambda}^{-1}, \lambda \in$ $\mathbb{R}_{+}$. A straightforward extension of the definition gives us symbols also in the case of Fréchet spaces with group action. A Green symbol of order $\nu$ associated with the weight data $(\gamma, \gamma-$ $m)$ is a $g(y, \eta) \in \bigcap_{s \in \mathbb{R}} S_{\mathrm{cl}}^{\nu}\left(U \times \mathbb{R}^{q} ; \mathcal{K}^{s, \gamma}\left(X^{\wedge}\right), \mathcal{S}^{\gamma-m+\varepsilon}\left(X^{\wedge}\right)\right)$ such that $g^{*}(y, \eta) \in \bigcap_{s \in \mathbb{R}} S_{\mathrm{cl}}^{\nu}(U \times$ $\left.\mathbb{R}^{q} ; \mathcal{K}^{s,-\gamma+m}\left(X^{\wedge}\right), \mathcal{S}^{-\gamma+\varepsilon}\left(X^{\wedge}\right)\right)$ for some $\varepsilon>0$ where $g^{*}$ means the pointwise formal adjoint of $g$ with respect to the $\mathcal{K}^{0,0}$-scalar product. The group action $\kappa=\tilde{\kappa}$ in this case is defined by

$$
\left(\kappa_{\lambda} u\right)(r, x)=\lambda^{(n+1) / 2} u(\lambda r, x), \lambda \in \mathbb{R}_{+} .
$$

We apply this here to an open set $\Omega \subseteq \mathbb{R}^{q}$. Moreover, a smoothing Mellin symbol is an operator function of the form

$$
m(y, \eta)=r^{-m} \omega(r[\eta]) \operatorname{op}_{M}^{\gamma-n / 2}(f)(y) \omega^{\prime}(r[\eta]) \in S_{\mathrm{cl}}^{m}\left(\Omega \times \mathbb{R}^{q} ; \mathcal{K}^{s, \gamma}\left(X^{\wedge}\right), \mathcal{K}^{s-m, \gamma-m}\left(X^{\wedge}\right)\right.
$$

for cut-off functions $\omega, \omega^{\prime}$ and an $f(y, z) \in C^{\infty}\left(\Omega, M_{R}^{-\infty}(X)\right)$ for $R \bigcap \Gamma_{(n+1) / 2-\gamma}=\emptyset$.

We do not carry out the details of the edge pseudo-differential operators. Let us only recall that those are (up to global smoothing operators and contributions far from the edge $Y$ ) locally close to $Y$ of the form $A=\mathrm{Op}_{y}(a)$ for symbols of the form (4.4). Then, apart from the corresponding symbol $\sigma_{0}(A)$ and its reduced variant $\tilde{\sigma}_{0}(A)$, we have the principal edge symbol

$$
\sigma_{1}(A)(y, \eta)=r^{-m} \mathrm{op}_{M}^{\gamma-n / 2}\left(h_{0}\right)(y, \eta)+\sigma_{1}(m+g)(y, \eta): \mathcal{K}^{s, \gamma}\left(X^{\wedge}\right) \rightarrow \mathcal{K}^{s-m, \gamma-m}\left(X^{\wedge}\right),
$$

$(y, \eta) \in \Omega \times\left(\mathbb{R}^{q} \backslash\{0\}\right)$ for $h_{0}(r, y, z, \eta):=\tilde{h}(0, y, z, r \eta)$ and $\sigma_{1}(m+g)(y, \eta)$ defined as the homogeneous principal components of the respective classical symbols $m(y, \eta)$ and $g(y, \eta)$, namely,

$$
\sigma_{1}(m)(y, \eta)=r^{-m} \omega(r|\eta|) \operatorname{op}_{M}^{\gamma-n / 2}(f)(y) \omega^{\prime}(r|\eta|) \text { and } \sigma_{1}(g)(y, \eta)=g_{(m)}(y, \eta) .
$$

The operators (4.6) belong to the cone algebra on the open stretched cone $X^{\wedge}$ for every $(y, \eta) \in$ $\Omega \times\left(\mathbb{R}^{q} \backslash\{0\}\right)$. As such they also have the principal symbolic structure of the cone calculus

$$
\sigma_{0}\left(\sigma_{1}(A)\right)(r, x, y, \rho, \xi), \tilde{\sigma}_{0}\left(\sigma_{1}(A)\right)(r, x, y, \rho, \xi), \sigma_{1}\left(\sigma_{1}(A)\right)(y, z)
$$

for every fixed $y$, also referred to as subordinate symbols (here the dependence on $\eta$ diappears). Ellipticity of an operator $A$ in the edge calculus means ellipicity both with respect to $\sigma_{0}$ and $\sigma_{1}$. What concerns $\sigma_{0}$ the condition consists of the standard ellipticity together with non-vanishing of $\tilde{\sigma}_{0}$ up to $r=0$. This has the consequence that the first two components of (4.7) are non-vanishing in the sense of the cone calculus and that (4.6) is also exit-elliptic on $X$ for every $\eta \neq 0$. Then, according to Theorem 1.3 (iii), (iv), the only remaining condition for the Fredholm property of (4.3) for the given weight $\gamma$ is the ellipticity of $\sigma_{1}(A)(y, \eta)$ with respect to $\sigma_{1}\left(\sigma_{1}(A)\right)$, cf. the third item in formula (4.7), namely,

$$
\sigma_{1}\left(\sigma_{1}(A)\right)(y, z)=h_{0}(0, y, z, 0)+f(y, z) \in C^{\infty}\left(\Omega, M_{R}^{m}(X)\right) .
$$

This function is automatically elliptic in the sense of the assumption in Corollary 2.2, for every fixed $y$. Now Corollary 2.2 tells us that we may pass to another $\tilde{f}(y, z) \in C^{\infty}\left(\Omega, M_{\tilde{R}}^{-\infty}(X)\right)$ such that when we form

$$
\tilde{a}(y, \eta)=r^{-m} \operatorname{op}_{M}^{\gamma-n / 2}(h)(y, \eta) \epsilon^{\prime}+(\tilde{m}+\tilde{g})(y, \eta)
$$


for $\tilde{m}(y, \eta)=r^{-m} \omega(r[\eta]) \operatorname{op}_{M}^{\gamma-n / 2}(\tilde{f})(y) \omega^{\prime}(r[\eta])$, (and, if desired, another Green symbol $\left.\tilde{g}(y, \eta)\right)$ the edge symbol of $\tilde{A}=\mathrm{Op}_{y}(\tilde{a})$

$$
\sigma_{1}(\tilde{A})(y, \eta)=r^{-m} \mathrm{op}_{M}^{\gamma-n / 2}\left(h_{0}\right)(y, \eta)+\sigma_{1}(\tilde{m}+\tilde{g})(y, \eta): \mathcal{K}^{s, \gamma}\left(X^{\wedge}\right) \rightarrow \mathcal{K}^{s-m, \gamma-m}\left(X^{\wedge}\right)
$$

is Fredholm for the fixed $y$, and by construction we have $A=\tilde{A}$ modulo such a Mellin (plus Green) operator.

Remark 4.1. (i) The homogeneity of $\sigma_{1}(\tilde{A})(y, \eta)$ in the sense

$$
\sigma_{1}(\tilde{A})(y, \lambda \eta)=\lambda^{m} \kappa_{\lambda} \sigma_{1}(\tilde{A})(y, \eta) \kappa_{\lambda}^{-1}, \lambda \in \mathbb{R}_{+},
$$

allows us to reduce the mappings to $\eta \in S^{*} \Omega$, the unit cosphere bundle of $\Omega$. Then, if we restrict the consideration to $S^{*} \bar{\Omega}_{0}$ for an open set $\Omega_{0} \subset \Omega$ containing the point $y, \bar{\Omega}_{0} \subset \Omega$ compact, such that (4.8) are Fredolm for all $y \in \bar{\Omega}_{0}$, we obtain an index element

$$
\operatorname{ind}_{S^{*} \bar{\Omega}_{0}} \sigma_{1}(\tilde{A}) \in K\left(S^{*} \bar{\Omega}_{0}\right),
$$

the $K$-group of $S^{*} \bar{\Omega}_{0}$. An analogue of a well-known topological obstruction of Atiyah, Bott, cf. [1] concerning the existence of Shapiro-Lopatinskij elliptic boundary conditions (that may be non-vanishing also in edge problems, here concerning edge conditions) is the relation

$$
\operatorname{ind}_{S^{*} \bar{\Omega}_{0}} \sigma_{1}(\tilde{A}) \in \pi^{*} K\left(\bar{\Omega}_{0}\right)
$$

where $\pi^{*}$ is induced by the bundle pull-back under the canonical projection $\pi: S^{*} \bar{\Omega}_{0} \rightarrow \bar{\Omega}_{0}$.

(ii) It can be proved that the condition (4.11) is independent of the choice of $\tilde{f}$ involved in $\tilde{m}(y, \eta)$ which makes (4.8) to a family of Fredholm operators, $y \in \bar{\Omega}_{0}$. Moreover, in this case we find $\tilde{f}$ and $\tilde{g}$ in such a way that (4.8) is a family of isomorphisms for all $y \in \bar{\Omega}_{0}$. In other words, the ellipticity condition on $\sigma_{1}(\cdot)$ in the edge calculus which requires isomorphisms without additional Shapiro-Lopatinskij elliptic edge conditions is natural in a discussion of the edge algebra as the whole.

Remark 4.2. Remark 4.1 has an immediate analogue for BVPs on a manifold with edge for operators with the transmission property at the smooth part of the boundary.

\section{References}

[1] M.F. Atiyah and R. Bott, The index problem for manifolds with boundary, Coll. Differential Analysis, Tata Institute Bombay, Oxford University Press, Oxford, 1964, pp. 175-186.

[2] L. Boutet de Monvel, Boundary problems for pseudo-differential operators, Acta Math. 126 (1971), 11-51.

[3] J. Cheeger, On the spectral geometry of spaces with cone-like singularities, Proc. Nat. Acad. Sci. U.S.A. 76 (1979), 2103-2106.

[4] D. Kapanadze and B.-W. Schulze, Crack theory and edge singularities, Kluwer Academic Publ., Dordrecht, 2003.

[5] V.A. Kondratyev, Boundary value problems for elliptic equations in domains with conical points, Trudy Mosk. Mat. Obshch. 16 (1967), 209-292.

[6] R.B. Melrose and G.A. Mendoza, Elliptic operators of totally characteristic type, Preprint MSRI 047 - 83, Math. Sci. Res. Institute, 1983. 
[7] B.-W. Schulze, Pseudo-differential operators on manifolds with singularities, North-Holland, Amsterdam, 1991.

[8] B.-W. Schulze, Boundary value problems and singular pseudo-differential operators, J. Wiley, Chichester, 1998.

[9] B.-W. Schulze, Operator algebras with symbol hierarchies on manifolds with singularities, Advances in Partial Differential Equations (Approaches to Singular Analysis) (J. Gil, D. Grieser, and Lesch M., eds.), Oper. Theory Adv. Appl., Birkhäuser Verlag, Basel, 2001, pp. 167-207.

[10] B.-W. Schulze, The iterative structure of corner operators; Lecture during the Conference "Elliptic and Hyperbolic Equations on Singular Spaces", October 27-29, 2008, at the MSRI, Berkeley, Preprint 2008/08, Institut für Mathematik, Potsdam, 2008.

[11] M.C. Shaw, Hodge theory on domains with conic singularities, Comm. Part. Diff. Equ. 8, 1 (1983), 65-88.

[12] I. Witt, On the factorization of meromorphic Mellin symbols, Advances in Partial Differential Equations (Parabolicity, Volterra Calculus, and Conical Singularities) (S. Albeverio, M. Demuth, E. Schrohe, and B.-W. Schulze, eds.), Oper. Theory Adv. Appl., vol. 138, Birkhäuser Verlag, Basel, 2002, pp. 279-306. 